\title{
Erratum: Naclerio et al. (2017)
}

In the article Naclerio, F., Seijo, M., Larumbe-Zabala, E., \& Earnest, C.P. (2017). Carbohydrates alone or mixing with beef or whey protein promote similar training outcomes in resistance training males: a double-blind, randomized controlled clinical trial. International Journal of Sport Nutrition and Exercise Metabolism, 27(5), 408-420, https://doi.org/10.1123/ijsnem.2017-0003, an author name was misspelled. Marcos Seijo was misspelled as Marco Seijo-Bujia. The online version of this article has been corrected. We apologize for this error. 